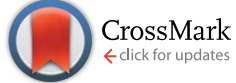

Cite this: RSC Adv., 2017, 7, 4348

Received 6th December 2016 Accepted 27th December 2016

DOI: $10.1039 / c 6 r a 27843 d$

www.rsc.org/advances

\section{Amphiphilic hyperbranched polyglycerols in a new role as highly efficient multifunctional surface active stabilizers for poly(lactic/glycolic acid) nanoparticles $\uparrow$}

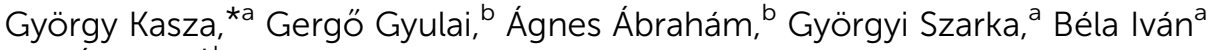 \\ and Éva Kiss ${ }^{* b}$
}

\begin{abstract}
Amphiphilic hyperbranched polyglycerols (HbPG) were synthesized by dodecyl and octadecyl alcohols as direct initiators for the ringopening multibranching polymerization of glycidol. These polymers possess molecular weight dependent surface activity, and are efficient surfactants and stabilizers for poly(lactic/glycolic acid) (PLGA) nanoparticles, opening new possibilities for functionalized drug carriers, targeting, imaging agents, etc.
\end{abstract}

Hyperbranched amphiphilic (co)polymers have recently gained significant interest due mainly to their unique structure and properties which make them suitable building blocks for a large variety of yet unexplored macromolecular architectures, including but not limited to self-assembled nanosystems or even nanoreactors. ${ }^{1}$ One of the most attractive candidates for such purposes is hyperbranched polyglycerol (HbPG), which is a polyether polyol with numerous advantageous properties, such as simple and controllable synthesis, outstanding water solubility and high stability. ${ }^{2}$ The physical and chemical properties of HbPG can be easily varied by derivatization through the high number of its hydroxyl functionalities, and thus it can be utilized as building blocks of complex macromolecular structures. ${ }^{3,4}$ Furthermore, HbPG is a biocompatible polymer as has been proved by several related observations, such as reduced protein adsorption, blood compatibility, non-immunogenic and non-toxic character. ${ }^{5}$ Therefore, not surprisingly, the structureproperty relations and application possibilities of this hyperbranched polymer have been widely investigated, especially in biomedical fields. The hydrophilic chain ends and the more

${ }^{a}$ Polymer Chemistry Research Group, Institute of Materials and Environmental Chemistry, Research Centre for Natural Sciences, Hungarian Academy of Sciences, Magyar tudósok körútja 2, H-1117 Budapest, Hungary. E-mail: kasza.gyorgy@ttk. mta.hu

${ }^{b}$ Laboratory of Interfaces and Nanostructures, Institute of Chemistry, Eötvös Loránd University, H-1518 Budapest 112, PO Box 32, Hungary. E-mail: kisseva@chem.elte.hu $\dagger$ Electronic supplementary information (ESI) available: Experimental procedures and characterization results of HbPG samples. Morphological characterization of PLGA nanoparticles. Protein adsorption on alkyl-HbPG covered surface, QCM measurement. See DOI: 10.1039/c6ra27843d hydrophobic space within the flexible chains provide many options for labelling and immobilizing bioactive components. Several HbPG based amphiphilic core-shell nanocarriers were described and successfully applied to increase the solubilization efficiency of drugs or dyes. ${ }^{6,12}$ Unimolecular micelles with HbPG core were synthesized via functionalization of the hydroxyl groups by hydrophobic moieties and/or linear polymers. ${ }^{7}$ Micellar drug carriers were also developed by utilizing hydrophobic initiators or macromolecules in the synthesis of HbPG and applied to produce self-assembled drug delivery nanocapsules. ${ }^{\mathbf{8} 12}$ Although different HbPGs with amphiphilic characteristics were synthesized, these materials have not been applied and investigated for the preparation and surface modification of polymeric nanoparticles (NPs) made from one of the most promising biocompatible and biodegradable polymer, poly(lactic/glycolic acid) (PLGA).

Herein, we report on a convenient synthetic route to prepare amphiphilic hyperbranched polyethers with one hydrophobic linear block and the hydrophilic HbPG segment. This welldefined structure is expected to be suitable for interfacial adsorption, and thus for forming a surface layer. Two simple aliphatic units, like dodecyl and octadecyl chains, were used as initiators, and the interfacial properties of the resulting HbPGs were investigated and compared. Our aim was to study whether these HbPG derivatives can be used as surface modifiers in the preparation of PLGA NPs. Such NPs produced from the biocompatible and biodegradable PLGA copolymers with tunable properties are suitable carriers for providing controlled release of a large variety of compounds. However, the low solubility and particle aggregation are significant limitations for the application of PLGA NPs. Thus, we aimed at studying the effect of amphiphilic HbPG, capable for subsequent functionalization, on the stabilization of PLGA NPs. The resulting PLGA-HbPG polymeric nanostructures might satisfy the requirements of multifunctional carrier systems for controlled drug release with simultaneous targeting and imaging possibilities.

Amphiphilic HbPGs were synthesized by bulk ring-opening multibranching polymerization of glycidol by the adaption of a previously described technique ${ }^{8}$ (see Experimental in the 
ESI $\dagger$ ). However, in contrast to previous work reporting on the use of modified hexadecyl amine as initiator, ${ }^{8}$ we found that the synthesis of HbPG by direct initiation with alkyl alcohols, i.e. with dodecyl (C12) and octadecyl (C18) alcohols, can be successfully carried out. The ${ }^{1} \mathrm{H}$ NMR analysis of the resulting polymers, i.e. C12-HbPG and C18-HbPG, proved the incorporation of the alkyl chains, and gave $M_{\mathrm{n}}=2690 \mathrm{~g} \mathrm{~mol}^{-1}$ and $M_{\mathrm{n}}=$ $3750 \mathrm{~g} \mathrm{~mol}^{-1}$, respectively (see ESI: Experimental and characterization details $\dagger$ ). The compact, highly branched structure of these polymers is also supported by the apparent $M_{\mathrm{n}}$ values $\left(1860 \mathrm{~g} \mathrm{~mol}^{-1}(\mathrm{PD}=1.32)\right.$ and $2210 \mathrm{~g} \mathrm{~mol}^{-1}(\mathrm{PD}=1.50)$ for C12-HbPG and C18-HbPG, respectively) determined by GPC calibrated with linear polymer standards. The $M_{\mathrm{n}} \mathrm{s}$ by GPC are significantly lower than that obtained by ${ }^{1} \mathrm{H}$ NMR spectroscopy (ESI, Fig. S1-S3 and Table S1 $\dagger$ ). This observation is attributed to the lower hydrodynamic volume of branched polymers than that of their linear counterparts. The hydrophobic content of the alkyl-HbPGs, calculated from the ${ }^{1} \mathrm{H}$ NMR results, are approximately equal $(6.9 \%$ for $\mathrm{C} 12-\mathrm{HbPG}$ and $7.2 \%$ for C18-HbPG), so these polymers can be satisfactorily utilized for comparative investigations on the effect of alkyl chain length and molecular weight in the preparation and stabilization of PLGA nanoparticles.

The surface activity of the alkyl-HbPGs was characterized by measuring the surface tension of their aqueous solution by drop shape analysis ${ }^{\mathbf{9} 10}$ (OCA15+Dataphysics, Germany) as a function of concentration in the range of $10^{-4}$ to $10^{2} \mathrm{~g} \mathrm{l}^{-1}$. The surface tension, plotted in Fig. 1, decreases even at low concentrations showing a strong tendency of surface adsorption of the HbPG molecules due to their amphiphilic nature. The shape of the curve is typical for a surfactant having decreasing surface tension with increasing concentration followed by reaching a low value which does not decrease further. As shown in Fig. 1, the critical micellar concentration ( $\mathrm{cmc}$ ) can be obtained by determining the intersection of the straight lines fitted to the data in the decreasing and lower plateau regions of the surface tension versus concentration plots. The cmc value is $2.6 \mathrm{~g} \mathrm{l}^{-1}$ $\left(1 \times 10^{-3} \mathrm{M}\right)$ for $\mathrm{C} 12-\mathrm{HbPG}$ and two orders of magnitudes lower for the C18-HbPG, $0.08 \mathrm{~g} \mathrm{l}^{-1}\left(2.1 \times 10^{-5} \mathrm{M}\right)$. The formation of micellar type aggregates above cmc is supported by DLS measurements as well (Brookhaven Instruments, USA). No

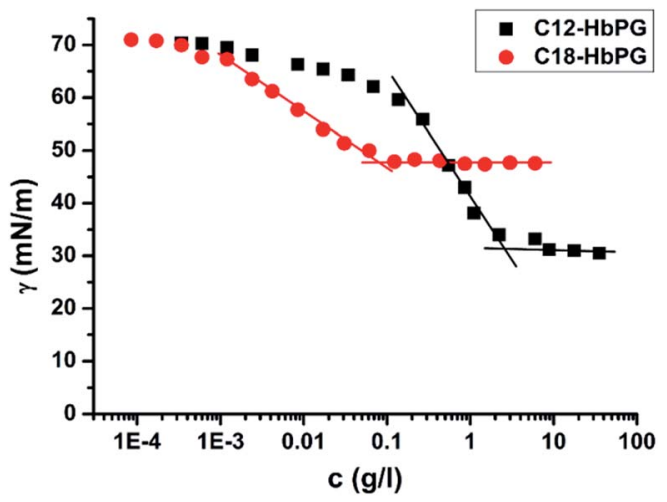

Fig. 1 Surface tension of aqueous solutions of alkyl-HbPGs as a function of concentration. aggregates were found in the alkyl-HbPG solutions below $\mathrm{cmc}$, but associates with hydrodynamic diameter in the range of 5-7 nm were detected above cmc. The cmc values obtained for C12-HbPG and C18-HbPG are consistent with that reported previously $^{8}$ for C16-HbPG10 $\left(5-20 \times 10^{-5} \mathrm{M}\right)$ determined by a dye solubilization type method. The comparison of the two curves in Fig. 1 indicates quite reasonably the higher surface activity of the C18-HbPG molecule with the longer alkyl chain, on the one hand. On the other hand, both the slope of the surface tension curves and the saturation values are different. This can be attributed to the different sizes of the HbPG molecules. The Gibbs-equation was used to calculate the surface excess of the surface active materials on the basis of the experimental $\gamma v s$. $c$ relation. There is a significant difference in these values for the two alkyl-HbPGs. The molecular area occupied by C12-HbPG in the saturated surface layer $\left(1.5 \mathrm{~nm}^{2}\right)$ is smaller, i.e. it forms a more dense adsorbed layer than that of the C18-HbPG with corresponding molecular area of $2.06 \mathrm{~nm}^{2}$. This relation is consistent with the molecular weights (see ESI, Table S1†) because the C18-HbPG contains not only a longer alkyl chain but also a larger HbPG component. It is important to note that the interfacial adsorption and micelle forming ability of the alkyl-HbPG molecules can be sensitively tuned by the length of the alkyl chains in impressively broad concentration ranges, similar to the traditional surfactants.

Highly hydrophilic surfaces, especially in the lack of electrostatic interaction, are known to show reduced protein adsorption which is considered as an essential requirement of surface biocompatibility. ${ }^{10}$ Poly(ethylene oxide- $b$-propylene oxide- $b$-ethylene oxide) block copolymers (Pluronics) are among those applied preferentially as surface modifiers for such purposes. ${ }^{11}$ HbPGs with highly hydrophilic character having flexible chains are notable alternatives for the formation of biocompatible coatings as reported in previous investigations. ${ }^{2 a, 5 e}$ Therefore, interaction of protein molecules with the adsorbed layer of alkyl-HbPGs was investigated in situ by quartz crystal microbalance technique. Bovine serum albumin adsorption on the surface of the sensor crystal was greatly reduced to approximately $20 \%$ compared to the pure gold surface, due to the preadsorbed C18-HbPG layer. The frequency change diagram with the Experimental details is presented in the ESI (Fig. S5 $\dagger$ ).

PLGA nanoparticles in the presence of HbPG were prepared by a nanoprecipitation method described previously. ${ }^{13-15}$ Briefly,

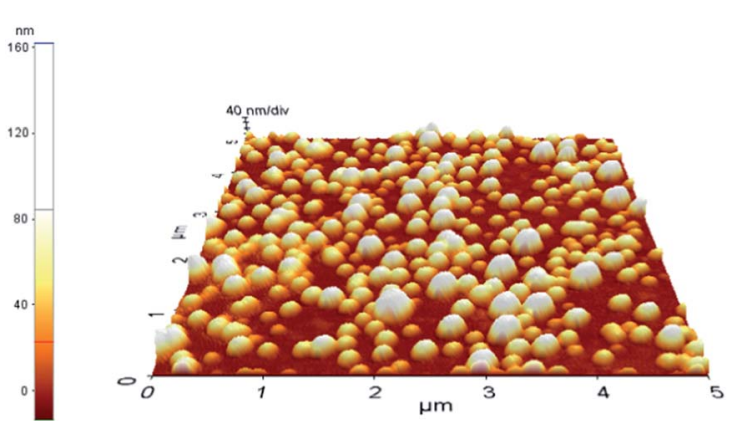

Fig. 2 AFM image of PLGA nanoparticles formed with the application of alkyl-HbPG stabilizer. 
PLGA was dissolved in acetone and added in a controlled way to aqueous solution of the alkyl-HBPG stabilizer. The resulting sol was purified by dialysis and repeated centrifugation to remove the excess of the dissolved components.

The atomic force microscopy (AFM) images of PLGA/alkylHbPG NPs show spherical particles with smooth surface and narrow size distribution (Fig. 2). The average hydrodynamic size and polydispersity (PD) of the PLGA NPs were determined by DLS. It was found that the alkyl-HbPG stabilizer has some influence on the size of PLGA nanoparticles in comparison with the previously used Pluronics. ${ }^{14}$ The average hydrodynamic diameter of the NPs stabilized by alkyl-HbPG was in the range of 110-120 nm with a PD of less than 0.1 in contrast to the size of 140-155 nm with similar polydispersity obtained for NPs using Pluronic127. The thickness of the adsorption layer of alkylHbPG is supposed to be thin, hence no effect of the applied concentration on the size was detectable.

The advantage of the surface modification by the alkyl-HbPG polymers is the improvement of surface hydrophilicity and biocompatibility of PLGA and the protection of the sol against aggregation. Therefore, in addition to the characterization of the particle size, the zeta-potential and colloidal stability were also investigated as a function of the concentration of alkylHbPGs. The measurements of the electrophoretic mobility of nanoparticles in aqueous salt solution (2 $\mathrm{mM} \mathrm{NaCl})$ were carried out by means of a Malvern Zetasizer 4 apparatus. ${ }^{15}$ Zetapotential of the nanoparticles as an indication of the composition of the particle surface was calculated by using the Smoluchowski approximation. The obtained values for C18-HbPG stabilized PLGA NP are displayed as a function of concentration in comparison with Pluronic stabilized particles in Fig. 3.

The PLGA particles in the absence of stabilizing polymer have negative surface charge since the carboxylic chain ends of the PLGA are preferably exposed at the surface while dispersed in the aqueous medium. Polymer adsorption (Pluronic or alkyl$\mathrm{HbPG}$ ) onto the surface was found to reduce the zeta potential in a concentration dependent manner. Significantly lower zetapotential values were obtained for both polymers as an indication of adsorption. The reason for this change in the zetapotential might be twofold. The adsorbed alkyl-HbPG layer

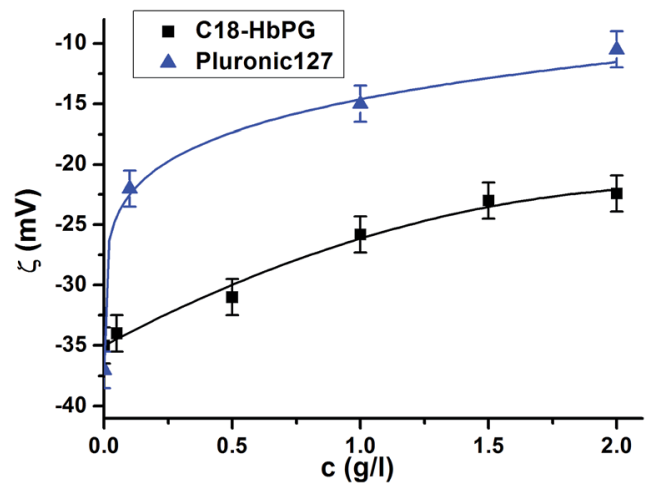

Fig. 3 Zeta-potential of PLGA nanoparticles stabilized by $\mathrm{C} 18-\mathrm{HbPG}$ in comparison with Pluronic127 stabilized PLGA as a function of polymer concentration. covering the surface can shield partly the terminal negative charges in the PLGAs. In addition, the presence of the hydrated polymer layer on the particle surface pushes the shear plane towards the medium decreasing further the zeta-potential. Presumably, the latter effect is responsible for the difference in the zeta-potential values at high surface coverage for the alkyl-HbPG stabilized nanoparticles. The thickness of the adsorbed layer is obviously lower for the alkyl-HbPG with molecular weight of about $3 \mathrm{~kg} \mathrm{~mol}^{-1}$ compared to Pluronic127 with $12 \mathrm{~kg} \mathrm{~mol}^{-1}$ having a different molecular architecture as well. Because of the presence of the hydrated polymer layer on the PLGA surface, the sol is expected to be sterically stabilized. This was estimated by coagulation experiments. The effect of ionic strength on the colloidal stability of alkyl-HbPG coated PLGA nanoparticles was investigated by turbidity measurements ${ }^{\mathbf{1 4 , 1 5}}$ by adding $\mathrm{NaCl}$ solution to the suspension with increasing concentration. The highest $\mathrm{NaCl}$ concentration which resulted in no coagulation is displayed in Fig. 4. As shown in this figure, C18-HbPG proved to be an efficient steric stabilizer in the whole studied concentration range. The other derivative with the shorter alkyl chain can stabilize the nanoparticles against electrolyte induced coagulation only by applying higher concentrations, i.e. above $1.5 \mathrm{~g} \mathrm{l}^{-1}$. This behavior is probably in connection with the adsorption strength of the amphiphilic polymer molecules. The adsorption is weaker for the alkyl-HbPG with the shorter alkyl chain.

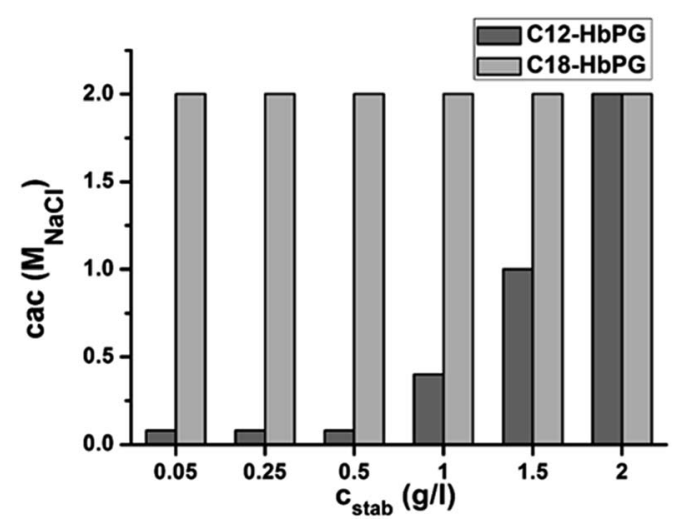

Fig. 4 Aggregation stability of the PLGA nanoparticles stabilized by alkyl-HbPGs characterized by the critical coagulation concentration.
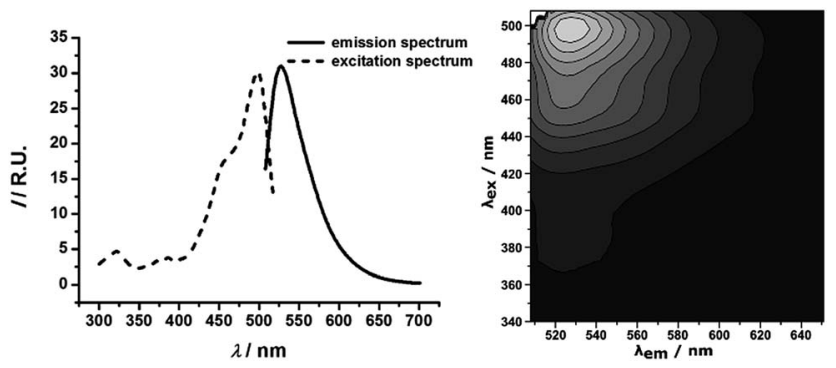

Fig. 5 Emission and excitation spectra (left) as well as the map of fluorescence intensity (right) of PLGA nanoparticles stabilized by fluorescently labelled C18-HbPG. 


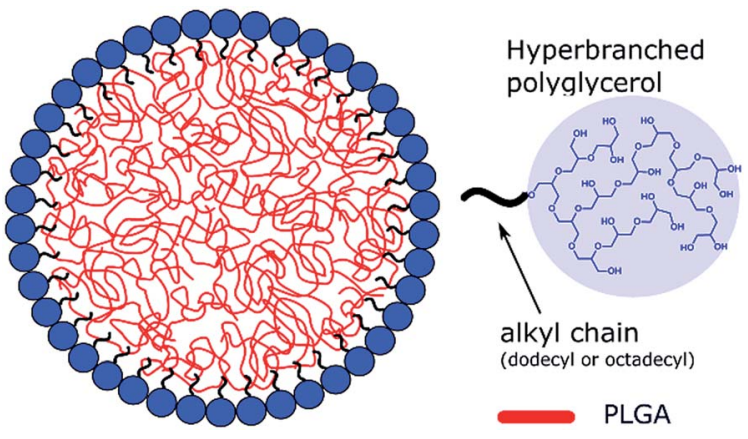

Fig. 6 The schematic structure of the alkyl-HbPG stabilized PLGA nanoparticle.

To demonstrate that this construction, i.e. the alkyl-HbPG stabilized PLGA, is suitable for the preparation of NPs with multifunctional surface, 5(6)-carboxyfluorescein (CF) was bonded chemically via an esterification reaction to the C18HbPG (see ESI $\dagger$ for Experimental details). Based on the ${ }^{1} \mathrm{H}$ NMR spectrum (Fig. S4 $\dagger$ ), the functionalization was successful, and the dye content is approximately one CF per HbPG macromolecule. The resulting fluorescent polymer was used in the stabilization of PLGA NPs. It was found that the labelling of the stabilizer has no influence on the formation, the size and stability of the alkyl-HbPG stabilized PLGA NPs giving an intense fluorescence as shown in Fig. 5 .

\section{Conclusions}

In sum, it can be concluded that hyperbranched polyglycerols with one alkyl chain (C12-HbPG and C18-HbPG) can be directly synthesized with the corresponding alcohols, and the resulting polymers can be successfully applied as surface modifiers and stabilizers in the preparation of PLGA nanoparticles. As depicted in Fig. 6, the amphiphilic HbPG covers the surface of the PLGA nanoparticles due to the orienting interaction of the hydrophobic alkyl segment of the alkyl-HbPG and PLGA surface as proved by the results of zeta-potential and aggregation experiments. This surface coverage by the amphiphilic HbPGs provides outstanding colloidal stability of the PLGA NPs. The HbPG surface layer, in comparison with the previously used Pluronics, offers unique advantages because the numerous hydroxyl groups of HbPG molecule can be modified easily by imaging, such as CF, and/or targeting moieties. Thereby, it is possible to develop a large variety of PLGA nanoparticles with (multi)functional surfaces by utilizing alkyl-HbPGs.

\section{Acknowledgements}

The authors are grateful for the technical assistance in GPC analyses by Mr Tamás Ignáth. Support by the MedInProt Protein Science Research Synergy/Mentor Program, the National Research, Development and Innovation Office, Hungary (OTKA K104928 and K112094) and the MTA Postdoctoral Research Program (G. Gy.) is also acknowledged.

\section{Notes and references}

1 (a) H. Chen and J. Kong, Polym. Chem., 2016, 7, 3643-3663; (b) H. Jin, W. Huang, X. Zhu, Y. Zhou and D. Yan, Chem. Soc. Rev., 2012, 41, 5986-5997; (c) I. Gitsov, I. V. Berlinova and N. G. Vladimirov, J. Polym. Sci., Part A: Polym. Chem., 2015, 53, 178-182; (d) W. Jiang, Y. Zhou and D. Yan, Chem. Soc. Rev., 2015, 44, 3874-3889; (e) I. Gitsov, J. Hamzik, J. Ryan, A. Simonyan, J. P. Nakas, S. Omori, A. Krastanov, T. Cohen and S. W. Tanenbaum, Biomacromolecules, 2008, 9, 804-811; $(f)$ W. W. Fan, X. D. Fan, W. Tian, X. Zhang, G. Wang, W. B. Zhang, B. Yang and X. Z. Zhu, Polym. Chem., 2014, 5, 4022-4031.

2 (a) C. Siegers, M. Biesalski and R. Haag, Chem.-Eur. J., 2004, 10, 2831-2838; (b) H. Frey and R. Haag, Rev. Mol. Biotechnol., 2002, 90, 257-267; (c) Y. Huang, D. Wang, X. Zhu, D. Yan and R. Chen, Polym. Chem., 2015, 6, 2794-2812.

3 (a) D. Haamann, H. Keul, D. Klee and M. Möller, Macromolecules, 2010, 43, 6295-6301; (b) M. Backes, L. Messager, A. Mourran, H. Keul and M. Moeller, Macromolecules, 2010, 43, 3238-3248; (c) C. Schmitz, H. Keul and M. Möller, Eur. Polym. J., 2009, 45, 2529-2539; (d) I. N. Kurniasih, J. Keilitz and R. Haag, Chem. Soc. Rev., 2015, 44, 4145-4164; (e) D. Wilms, S.-E. Stiriba and H. Frey, Acc. Chem. Res., 2010, 43, 129-141; (f) J. T. Sun, C. Y. Hong and C. Y. Pan, Polym. Chem., 2011, 2(5), 998-1007.

4 (a) M. Schömer, J. Seiwert and H. Frey, ACS Macro Lett., 2012, 1, 888-891; (b) E. M. Christ, D. Hobernik, M. Bros, M. Wagner and H. Frey, Biomacromolecules, 2015, 16, 32973307; (c) A. Utrata-Wesołek, N. Oleszko, B. Trzebicka, J. Anioł, M. Zag-dańska, M. Lesiak, A. Sieron and A. Dworak, Eur. Polym. J., 2013, 49, 106-117; (d) A. UtrataWesołek, W. Wałach, J. Anioł, A. L. Sieroń and A. Dworak, Polymer, 2016, 97, 44-54.

5 (a) R. K. Kainthan, J. Janzen, E. Levin, D. V. Devine and D. E. Brooks, Biomacromolecules, 2006, 7, 703-709; (b) R. K. Kainthan, M. Gnanamani, M. Ganguli, T. Ghosh, D. E. Brooks, S. Maiti and J. N. Kizhakkedathu, Biomaterials, 2006, 27, 5377-5390; (c) A. Sunder, R. Mülhaupt, R. Haag and H. Frey, Adv. Mater., 2000, 12, 235-239; (d) M. Imran ul-haq, B. F. Lai, R. Chapanian and J. N. Kizhakkedathu, Biomaterials, 2012, 33, 9135-9147; (e) M. Calderón, M. A. Quadir, S. K. Sharma and R. Haag, Adv. Mater., 2010, 22, 190-218; (f) P. Y. J. Yeh, R. K. Kainthan, Y. Zou, M. Chiao and J. N. Kizhakkedathu, Langmuir, 2008, 24, 4907-4916.

6 (a) M. Gosecki, M. Gadzinowski, M. Gosecka, T. Basinska and S. Slom-kowski, Polymers, 2016, 8, 227; (b) M. Libera, P. Formanek, L. Schellkopf, B. Trzebicka, A. Dworak and M. Stamm, J. Polym. Sci., Part A: Polym. Chem., 2014, 52, 3488-3497.

7 (a) R. Haag, J. F. Stumbé, A. Sunder, H. Frey and A. Hebel, Macromolecules, 2000, 33, 8158-8166; (b) S. Xu, Y. Luo, R. Graeser, A. Warnecke, F. Kratz, P. Hauff and R. Haag, Bioorg. Med. Chem. Lett., 2009, 19, 1030-1034; (c) I. N. Kurniasih, H. Liang and J. P. Rabe, Macromol. Rapid 
Commun., 2010, 31, 1516-1520; (d) C. Mugabe, Y. Matsui, A. I. So, M. E. Gleave, M. Heller, M. Zeisser-Labouèbe, L. Heller, I. Chafeeva, D. E. Brooks and H. M. Burt, Biomacromolecules, 2011, 12, 949-960; (e) S. Nowag, C. Frangville, G. Multhaup, J. D. Marty, C. Mingotaud and R. Haag, J. Mater. Chem. B, 2014, 2, 3915-3918; $(f)$ R. Knischka, J. P. Lutz, A. Sunder, R. Mülhaupt and H. Frey, Macromolecules, 2000, 33, 315-320; $(g)$ C. S. Popeney, M. C. Lukowiak, C. Böttcher, B. Schade, P. Welker, D. Mangoldt, G. Gunkel, Z. Guan and R. Haag, ACS Macro Lett., 2012, 1(5), 564-567.

8 V. Istratov, H. Kautz, Y. K. Kim, R. Schubert and H. Frey, Tetrahedron, 2003, 59, 4017-4024.

9 É. Kiss, E. T. Heine, K. Hill, Y. C. He, N. Keusgen, C. B. Pénzes, D. Schnöller, G. Gyulai, A. Mendrek, H. Keul and M. Moeller, Macromol. Biosci., 2012, 12, 1181-1189.

10 (a) É. Kiss, E. Kutnyánszky and I. Bertóti, Langmuir, 2010, 26, 1440-1444; (b) É. Kiss, Colloids Surf., A, 1993, 76, 135-140.
11 (a) É. Kiss, M. G. Takács, I. Bertóti and E. I. Vargha-Butler, Polym. Adv. Technol., 2003, 14, 839-846; (b) G. Gyulai, C. B. Pénzes, M. Mohai, T. Lohner, P. Petrik, S. Ku-runczi and É. Kiss, J. Colloid Interface Sci., 2011, 362, 600-606.

12 (a) T. V. Demina, O. A. Budkina, G. A. Badun, N. S. MelikNubarov, H. Frey, S. S. Müller, J. Nieberle and I. D. Grozdova, Biomacromolecules, 2014, 15, 2672-2681; (b) A. Richter, A. Wiedekind, M. Krause, T. Kissel, R. Haag and C. Olbrich, Eur. J. Pharm. Sci., 2010, 40, 48-55.

13 É. Kiss, D. Schnöller, K. Pribranska, K. Hill, C. B. Pénzes, K. Horváti and S. Bősze, J. Dispersion Sci. Technol., 2011, 32, 1728-1734.

14 G. Gyulai, C. B. Pénzes, M. Mohai, F. Csempesz and É. Kiss, Eur. Polym. J., 2013, 49, 2495-2503.

15 É. Kiss, G. Gyulai, C. B. Pénzes, M. Idei, K. Horváti, B. Bacsa and S. Bősze, Colloids Surf., A, 2014, 458, 178-186. 